\title{
Representações Sociais da AIDS, Práticas Sexuais e Vida Social entre Heterossexuais, Bissexuais e Homossexuais em Brasília, Brasil
}

\author{
Social Representations Concerning AIDS, Sexual Practices and Social \\ Life among Heterosexuals, Bisexuals and Homosexuals in Brasilia, \\ Brazil
}

\author{
Edson A. de Souza Filho \\ Márcia G. Henning ${ }^{1}$
}

SOUZA-FILHO, E. A. \& HENNING, M. G. Social Representations Concerning AIDS, Sexual Practices and Social Life among Heterosexuals, Bisexuals and Homosexuals in Brasilia, Brazil. Cad. Saúde Públ., Rio de Janeiro, 8 (4): 428-441, oct/dec, 1992.

The social representations of AIDS, preventive practices concerning sexually transmissible diseases, self-representations, social life, values, and forms of information for 89 heterosexuals $(H E), 7$ bisexuals $(B I)$ and 33 homosexuals $(H O)$ were investigated through a questionnaire containing 33 open and closed questions. Results showed a general belief that everybody is vulnerable to the AIDS virus, but that external changes in the body were considered the major sympton of AIDS by HE and HO. Vaginal sex (30.47\%) and oralyanal sex (43.33\%) were the most commonly referred by $H E$ and $H O$, respectively, while $B I$ were more diffuse with regard to their sexual practices. In addition to the use of condoms, $H E$ referred avoiding risk groups and opting for only one partner, while $H O$ would avoid sex (18.2\%). Despite $H E$ and $H O$ selfrepresentations in terms of sexual behaviour they gave more emphasis to family than to sexual activity - something that could be regarded as an indication of psychosocial change in these groups caused by AIDS. Suggestions for future research included BI practices as a link between the groups, the power and trust relationships among HE partners, and the urgent need for further studies on the psycho-social life of these groups.

Keywords: AIDS, Social Representation; Sexual Behavior; Social Psychology

\section{INTRODUÇÃO}

Desde o seu aparecimento, em 1981, que a AIDS vem atraindo uma atenção cada vez maior do público mundial, já que se trata de uma epidemia que ameaça a todos (Herzlich \& Pierret, 1988), para a qual, ainda no final da década, não havia sido encontrada uma forma de tratamento e prevenção que anulasse, de forma definitiva, seus efeitos e alastramento.

Sabemos que entre as formas de contágio

\footnotetext{
' Departamento de Psicologia Social e do Trabalho da Fundação Universidade de Brasilia, 79910-900, Brasília, DF, Brasil.
}

mais importantes está a via sexual. Nesse sentido, alguns estudos sugeriram que as práticas anal e oral estariam difundidas entre heterossexuais (Parker, 1987). Outras observações davam conta do pouco ou nenhum uso de preservativo entre todos os grupos sexuais no Brasil (Adeodato, 1991; Gunther et al., 1991; Martin et al., 1991). Na mesma linha de raciocínio, a relativa marginalização de homossexuais e bissexuais masculinos na sociedade os tomaria possivelmente mais vulneráveis a práticas e concepções intragrupais (Pollak, 1987, 1988; Prieur, 1990). No caso da França, Pollak mostrou que, entre homossexuais masculinos, a concepção de prática sexual como "natural" (i.e., ligada à conduta sexual espontâ- 
nea) costumava estar associada à ausência de uso de técnicas preventivas em comportamento sexual e a uma maior submissão à pressão grupal para partilhar tais práticas e concepções. Segundo o mesmo autor, isso aconteceria mais entre os que tinham menos instrução e viviam em ambientes sociais intolerantes à homossexualidade, ao contrário dos que mantinham uma concepção de sexualidade como "racional" (i.e., com autocontrole em conduta sexual), que possuíam mais instrução e viviam em ambientes sociais mais tolerantes à homossexualidade. Estes últimos grupos haviam sido os primeiramente atingidos pela epidemia, facilitada por práticas "promíscuas" (Pollak, 1987), mas, logo em seguida, mais atentos à informação e à adoção de novas práticas preventivas.

No momento em que pensávamos em realizar esta pesquisa, pouco se sabia no Brasil sobre os aspectos psicossociais e sexuais em torno da AIDS. Era necessário, portanto, um aprofundamento psicossocial das várias dimensões acima referidas, ou seja, de um estudo exploratório a respeito do conhecimento da AIDS, de práticas sexuais e preventivas, e da vida social de heterossexuais, bissexuais e homossexuais.

A partir da problemática descrita em linhas gerais, consideramos adequada a adoção de um referencial teórico-metodológico mais aberto, que levasse em conta, simultaneamente, os aspectos simbólicos e sociais envolvidos, tal como o enfoque de representações sociais proposto por Serge Moscovici (1978).

Por representação social entende-se um saber informal originado no dia a dia para lidar com um assunto, pessoa ou objeto, quando este é estranho, abstrato ou ambíguo, a fim de torná-lo familiar, garantir comunicação univoca no interior do grupo, e interagir com outros individuos e grupos externos. Contrariamente aos modelos psicossociológicos que costumam encarar o tratamento de informação como um processo sobretudo individual, a proposta moscoviciana pretende situá-lo no interior de grupos e da sociedade, sem os quais não se pode compreender adequadamente os processos de circulação, assimilação ou indiferença em relação à informação (Echabe \& Rovira, 1989).

Para tanto, o modelo teórico proposto pelo mesmo autor apresenta algumas dimensões, nos núveis simbólico e social, que poderiam servir de parâmetros para a nossa investigação. No plano simbólico, considera todas as possibilidades de produção de significado de um sujeito a respeito de um assunto ou objeto qualquer, o que inclui a atitude em relação ao mesmo e leva em consideração as relações entre os significados mencionados, ou a constituição do campo de representação. Já no plano da interação social, Moscovici propôs três determinantes principais para a produção de representações sociais (Herzlich, 1972): a pressão à inferência exercida pelos grupos junto a seus membros, no sentido de tomar posição ou elaborar um saber informal a respeito de um assunto ou objeto importante para o funcionamento do grupo - para atingir metas, por exemplo; a focalização sobre o assunto ou objeto segundo o seu interesse ou situação social vivida grupalmente, não necessariamente consciente, como é o pertencer objetivamente a um grupo sexual (cf. Fry, 1972); e a informação tomada objetivamente sobre $o$ assunto ou objeto, sendo desigualmente distribuída na sociedade e determinando a subjetividade de um grupo particular a esse respeito.

Sabemos que algumas categorias de orientação sexual, tais como bissexuais e homossexuais, não chegam a constituir-se em grupos sociológicos no sentido convencional do termo, não possuindo, muitas vezes, aquelas estruturas grupais que os caracterizam. Sem pretendermos aprofundar a discussão, que já foi colocada alhures, ainda que de outro modo (Touraine, 1984), gostariamos de dizer que adotamos aqui uma noção de grupo sexual mais limitada, como o sentimento ou auto-representação de indivíduos que indicam práticas sexuais especificas.

A fim de orientar nossas observações, apresentamos abaixo os objetivos desta pesquisa:

1) Caracterizar o conhecimento sobre a AIDS em termos de formas de contágio, grupos vulneráveis, sintomas, origens, modos de diagnóstico, tratamento;

2) Caracterizar práticas sexuais de sujeitos que se autodefiniram como sendo heterossexuais, bissexuais ou homossexuais;

3) Caracterizar práticas preventivas em termos de número de parceiros, uso de preservativo, estratégias de prevenção, teste anti-HIV; 
4) Caracterizar a vida social de grupos sexuais em termos de identidade sexual, valores e participação social.

5) Caracterizar reações pessoais esperadas no caso de infecção de um amigo, de infecção de si mesmo, relevância social da epidemia, meios e locais de informação adequados para informar sobre a AIDS.

\section{METODOLOGIA}

Participaram da pesquisa basicamente três grupos, formados a partir de sua prática sexual declarada, ou seja, sujeitos que se identificaram como sendo heterossexuais (HE), bissexuais (BD) ou homossexuais (HO). A amostra de sujeitos que participaram da investigação não pretende a representatividade para os universos estudados, mas apenas revelar tendências psicossociais de microgrupos a serem cotejados com outros estudos.

No caso dos heterossexuais, estes foram contatados em função do acesso facilitado pela direção de uma escola de $2^{2}$ grau de Brasilia. Já a maioria dos bissexuais e homossexuais era freqüentadora de duas casas noturnas, também de Brasilia, cujo acesso nos foi facilitado por suas gerências.

Trabalhamos com um questionário elaborado por nós, parcialmente inspirado pelos utilizados por Henning \& Tamayo (1989) e Pollak (1988), contendo perguntas a respeito da identidade sexual, participação social, valores, conhecimento sobre a AIDS, práticas sexuais e preventivas, e comunicação social sobre o assunto. Ao todo, trabalhamos com 33 questões, abertas e fechadas.

A aplicação do questionário, realizada $\mathrm{em}$ maio de 1989, foi combinada com a direção da escola para ocorrer coletivamente, no intervalo de atividades curriculares. Na ocasião, estagiários de psicologia e um dos autores expuseram as intenções da pesquisa e pediram a colaboração dos estudantes no preenchimento dos questionários, os quais foram depositados em urnas fechadas. Gastou-se, em média, uma hora para fazê-lo, e houve adesão da maioria dos presentes à proposta.

Prevendo dificuldades conhecidas com os grupos bissexuais e homossexuais, adotamos um procedimento diferente. Inicialmente, a partir de agosto de 1989, com o apoio e autorização do gerente de uma casa noturna de Brasilia freqüentada por homossexuais masculinos, solicitamos o preenchimento de questionários a partir de envelopes selados, deixados com um empregado do estabelecimento, a serem recolhidos voluntariamente e devolvidos diretamente pelo correio a um dos autores. Como a adesão foi extremamente reduzida (dois questionários), apesar da existência de vários cartazes afixados no local, decidimos mudar o procedimento e usar o sistema de "redes de afinidades", ou seja, através de um dos gerentes de outra casa noturna da cidade, que distribuiu o questionário entre os seus amigos.

A análise de conteúdo das questões abertas aplicadas inclui a categorização das respostas, as quais foram objeto de análises estatísticas descritiva e inferencial, para comparação entre os grupos. Para descrevermos os sujeitos em termos de variáveis biodemográficas e sócio-culturais, empregamos apenas percentagens, e para estabelecermos inferências estatísticas, utilizamos o teste não-paramétrico KruskalWallis.

\section{RESULTADOS}

Das 33 questões aplicadas, apresentaremos, nesta publicação, apenas uma parte dos resultados obtidos.

Os grupos sexuais podem ser descritos em termos das variáveis sexo, idade, cidade de origem, escolaridade, estado civil, segundo os dados abaixo:

Às amostras incluíram $89 \mathrm{HE}$ (33 homens e 56 mulheres), 8 BI ( 7 homens e 1 mulher) e 33 HO (32 homens e 1 mulher).

Os HE apresentaram idades que variaram entre 15 e 21 anos $(66,3 \%), 22$ e $30(19,1 \%)$, e mais de $31(10,1 \%)$, enquanto $4,5 \%$ não responderam ao item. As idades dos BI variaram entre 15 e 21 ( 1 pessoa), 22 e 30 (6 pessoas), e 31 em diante ( 1 pessoa). Já entre os HO, 3 pessoas situaram-se entre 15 e 21 anos, 19 entre 22 e 30 anos, e 10 com mais de 31 anos, sendo que 1 não respondeu a este item.

Em termos de escolaridade, $88 \mathrm{HE}$ situaramse na faixa de $2 \%$ grau e 1 em nível superior. 
Entre os BI, por sua vez, 3 eram de $2^{2}$ grau, 2 de nivel superior, e 3 não especificaram. Já os HO, 1 era da faixa de $1^{9}$ grau, 8 de $2^{2}$ grau, 17 de nível superior, e 7 não especificaram.

Quanto ao estado civil dos grupos, obtivemos as seguintes informações: dos $\mathrm{HE}, 65$ sujeitos (73\%) eram solteiros, 20 casados $(22,5 \%), 2$ separados $(2,2 \%)$, e 2 não especificaram; dos BI, 6 sujeitos eram solteiros, 1 casado, e 1 separado; dos HO, finalmente, 29 eram solteiros, 2 casados, e 2 separados.

Gostariamos de esclarecer que o conhecimento informal, tal como reportado nas várias tabelas abaixo, é o resultado de análises de conteúdo de questões abertas aplicadas às populações (Bardin, 1979). Assim, as categorias simbólicas utilizadas por nós procuraram sintetizar de maneira objetiva e direta, o significado das respostas. Tais categorias encontradas podem ser consideradas como orientações simbólicas populares que devem ser levadas em conta no trabalho de preparação de campanhas de saúde pública e no atendimento clínico rotineiro. Assim sendo, ao leitor não habituado com este tipo de trabalho, a informação resul- tante exposta nas tabelas pode parecer, à primeira vista, simples e incompleta, como costuma se apresentar o senso comum a respeito de certos assuntos, os quais são tratados diferentemente por outros grupos sociais, tais como as comunidades profissionais e cientificas. Trata-se de relatar a sua especificidade de modo "quasedescritivo" para o que só exemplos concretos de como foram manifestados no discurso poderiam acrescentar informação, o que sobrecarregaria ainda mais o texto. Todos os dados simbólicos foram analisados estatisticamente, comparando os três grupos através do teste Kruskal-Wallis, com excessão dos referentes às práticas sexuais. Assim, as tabelas apresentadas adiante mostram os resultados das médias obtidas por cada grupo em cada tipo de resposta, sendo indicados, ademais, os niveis de significância de 'p'.

Em relação aos conteúdos de conhecimento sobre a AIDS segundo os grupos, estes foram ordenados nas Tabelas de 1 a 5 , apresentadas a seguir.

As práticas sexuais, à época do estudo, apontadas pelos sujeitos estão demonstradas na Tabela 6.

TABELA 1. Conhecimento sobre a Transmissão do HIV entre Heterossexuais, Bissexuais e Homossexuais

\begin{tabular}{lrrrrrr}
\hline \hline & \multicolumn{2}{c}{ HE } & \multicolumn{2}{c}{ BI } & \multicolumn{2}{c}{ HO } \\
& $\bar{y}$ & $\%$ & $\bar{x}$ & $\%$ & $\bar{x}$ & $\%$ \\
\hline Sexual & 0.92 & 86,5 & 0.62 & 62,5 & & 84,8 \\
Sangue & 0.53 & 48,0 & 0.25 & 25,0 & 0.78 & 69,7 \\
Droga injetável & 0.28 & 26,9 & & & 0.24 & 24,0 \\
Material hospitalar & 0.15 & 13,5 & & & 0.33 & 30,3 \\
Falta de informação & 0.14 & 13,5 & 0.12 & 12,5 & 0.09 & 9,1 \\
Objetos pessoais & 0.10 & 9,0 & & & 0.03 & 3,0 \\
Beijo, mosquito & 0.05 & 5,6 & & & 0.06 & 6,10 \\
Ferida & 0.04 & 4,5 & & & & \\
Destino & 0.02 & 2,2 & 0.12 & 12,5 & 0.03 & 3,0 \\
Debilidade orgânica & 0.01 & 1,1 & & & 0.03 & 3,0 \\
Outros & 0.13 & 13,5 & 0.37 & 37,5 & 0.21 & 18,2 \\
\hline \hline
\end{tabular}

${ }^{*} \mathrm{p} \leq 0.026$ (Teste Kruskal-Wallis) 
TABELA 2. Grupos que Podem Ser Atingidos pelo HIV

\begin{tabular}{lrrrrrr}
\hline \hline & \multicolumn{2}{c}{ HE } & \multicolumn{3}{c}{ BI } & \multicolumn{2}{c}{ HO } \\
& $\bar{x}$ & $\%$ & $\bar{x}$ & $\%$ & $\bar{x}$ & $\%$ \\
\hline Todos & 0.76 & 75,3 & 0.87 & 87,5 & 0.93 & 93,9 \\
Sexual & 0.32 & 32,0 & 0.37 & 37,5 & 0.36 & 36,4 \\
Hemofúlicos & 0.17 & 18,0 & & & 0.27 & 27,3 \\
Sexo desviante & 0.16 & 15,0 & 0.12 & 12,5 & 0.09 & 9,1 \\
Drogados & 0.12 & 12,4 & & & 0.12 & 12,1 \\
Não sabe & 0.04 & 4,5 & & & 0.03 & 3,0 \\
Outros & 0.23 & 22,4 & & & 0.15 & 15,2 \\
\hline \hline
\end{tabular}

TABELA 3. Sintomas da AIDS

\begin{tabular}{lcrrrrr}
\hline \hline & \multicolumn{2}{c}{ HE } & \multicolumn{3}{c}{ BI } & \multicolumn{3}{c}{ HO } \\
& $\bar{x}$ & \multicolumn{1}{c}{$\%$} & $\bar{x}$ & $\%$ & $\bar{x}$ & $\%$ \\
\hline Alterações corporais externas & 1.47 & 73,0 & 1.0 & 87,5 & 1.48 & 63,6 \\
Alterações corporais internas & 0.80 & 53,9 & 1.12 & 78,0 & 1.18 & 69,7 \\
Infeç̧ões & 0.58 & 48,2 & 0.62 & 62,5 & 1.09 & 63,6 \\
Alterações psicológicas & 0.06 & 6,7 & & & 0.15 & 15,2 \\
Outros & 0.30 & 30,3 & & & 0.21 & 21,2 \\
\hline \hline
\end{tabular}

${ }^{*} \mathrm{p} \leq 0.06$

TABELA 4. Origens do Vírus da AIDS

\begin{tabular}{|c|c|c|c|c|c|c|}
\hline & \multicolumn{2}{|c|}{$\mathrm{HE}$} & \multicolumn{2}{|c|}{ BI } & \multicolumn{2}{|c|}{ HO } \\
\hline & $\bar{x}$ & $\%$ & $\bar{x}$ & $\%$ & $\bar{x}$ & $\%$ \\
\hline Não sabe & 0.32 & 30,3 & & & 0.36 & 36,4 \\
\hline De laboratório & ${ }^{*} 0.28$ & 26,9 & 0.62 & 50,0 & 0.60 & 51,5 \\
\hline Doença sexual & 0.21 & 19,1 & 0.37 & 37,5 & 0.009 & 9,1 \\
\hline Já existia & 0.05 & 5,6 & & & 0.12 & 9,1 \\
\hline Droga & 0.02 & 2,2 & & & 0.06 & 3,0 \\
\hline Outros & 0.30 & 29,2 & 0.25 & 25,0 & 0.24 & 21,2 \\
\hline
\end{tabular}


TABELA 5. Tratamento da AIDS

\begin{tabular}{lrrrrrr}
\hline \hline & \multicolumn{2}{c}{ HE } & \multicolumn{2}{c}{ BI } & \multicolumn{2}{c}{ HO } \\
& $\bar{x}$ & $\%$ & $\bar{x}$ & $\%$ & $\bar{x}$ & $\%$ \\
\hline Paliativo & 0.35 & 31,4 & 0.25 & 25,0 & 0.66 & 63,6 \\
Sem tratamento & 0.32 & 32,6 & 0.25 & 25,0 & 0.21 & 21,2 \\
Não sabe & $* .15$ & 15,7 & 0.50 & 50,0 & & \\
Estão estudando & 0.08 & 9,0 & & & 0.09 & 9,1 \\
Depende de dinheiro & 0.07 & 7,9 & & & 0.06 & 6,1 \\
Fitoterapia e dieta & 0.02 & 2,2 & & & 0.06 & 6,1 \\
Depende da pessoa & 0.01 & 1,1 & & & 0.03 & 3,0 \\
Outros & 0.16 & 16,8 & 0.12 & 12,5 & 0.15 & 15,2 \\
\hline \hline
\end{tabular}

${ }^{*} \mathrm{p} \leq 0.024 ; \quad$ "* $\mathrm{p} \leq 0.001$

TABELA 6. Práticas Sexuais de Heterossexuais (HE), Bissexuais (BI) e Homossexuais (HO) dos Sexos Masculino (M) e Feminino (F)

\begin{tabular}{|c|c|c|c|c|c|c|}
\hline & \multicolumn{2}{|c|}{$\mathrm{HE}$} & \multicolumn{2}{|c|}{ BI } & \multicolumn{2}{|c|}{ HO } \\
\hline & $\begin{array}{l}\mathrm{M} \\
\%\end{array}$ & $\begin{array}{l}F \\
\%\end{array}$ & $\begin{array}{l}\mathbf{M} \\
\%\end{array}$ & $\underset{\text { \% }}{\mathrm{F}}$ & $\begin{array}{l}\text { M } \\
\%\end{array}$ & $\begin{array}{c}F \\
\%\end{array}$ \\
\hline Só sexo vaginal & 26,6 & 34,28 & 14,28 & & & \\
\hline Só sexo oral & & & 14,28 & & & \\
\hline Só sexo anal & & & & & 6,6 & \\
\hline Só masturbação mútua & & & 14,28 & & & 100,0 \\
\hline Só masturbação individual & 6,66 & 2,85 & & & 13,3 & \\
\hline Sexo vaginal c/s masturbação & 11,11 & 5,71 & & & & \\
\hline Sexo oral c/s masturbação & & & 14,28 & & 6,6 & \\
\hline Sexo anal c/s masturbação & & & & & 10,0 & \\
\hline Vaginal e oral c/s masturbação & 24,44 & 20,0 & 28,57 & 100,0 & & \\
\hline Vaginal e anal c/s masturbação & 6,66 & 5,71 & & & & \\
\hline Oral e anal c/s masturbação & & & & & 43,33 & \\
\hline Vaginal, oral e anal c/s masturbação & 11,11 & 5,71 & 14,11 & & 6,66 & \\
\hline Masturbação individual e mútua & 2,22 & & & & 13,33 & \\
\hline Nenhuma prática acima & 11,11 & 25,71 & & & & \\
\hline Total & 100,0 & 100,0 & 100,0 & 100,0 & 100,0 & 100,0 \\
\hline
\end{tabular}

O uso de técnicas preventivas pelos grupos em foco foi investigado a partir de algumas questões, cujos resultados podem ser lidos nas Tabelas de 7 a 10, expostas a seguir:

Quando perguntamos aos sujeitos se se submeteriam ao teste-AIDS, caso pudessem, $61,8 \%$ dos $\mathrm{HE}$ responderam afirmativamente, enquanto $22,5 \%$, negativamente. Já todos os BI e $51,5 \%$ dos HO declararam-se favoráveis ao teste, enquanto $15,2 \%$ destes últimos foram desfavo- ráveis. Nesse sentido, as justificativas apresentadas pelos que concordaram em se submeter ao teste se distribuíram da seguinte forma: por segurança: $24,7 \%$ (HE), 51,5\% (HO), os quais se diferenciaram quase que significativamente ( $\mathrm{p}<.061$ ); todos correm risco: 13,5 (HE), 12,5\% (HO); para não contaminar outros: $9 \%$ (HE) $21,1 \%$ (HO), sendo que $15,7 \%$ dos HE e $3 \%$ dos $\mathrm{HO}$ não especificaram a resposta. 
TABELA 7. Número de Parceiros Sexuais Ocasionais Tidos nos Últimos Três Meses

\begin{tabular}{llrc}
\hline \hline & $\mathrm{X}$ & $\mathrm{N}$ & $\%$ \\
\hline HE & 0,88 & 24 & 32,6 \\
BI & 0,34 & 5 & 75,0 \\
HO & 2,18 & 22 & 64,6 \\
\hline \hline
\end{tabular}

${ }^{*} \mathrm{p} \leq 0,002$
TABELA 8. Número de Vezes que Deixou de Usar Preservativo nos Últimos Três Meses

\begin{tabular}{llrl}
\hline \hline & $\mathrm{X}$ & $\mathrm{N}$ & $\%$ \\
\hline HE & 0,26 & 13 & 14,6 \\
BI & 0,50 & 2 & 25,0 \\
HO & 0,75 & 10 & 30,2 \\
\hline \hline
\end{tabular}

$* \mathrm{p} \leq 0,032$

TABELA 9. Estratégias Adotadas para Evitar o HIV

\begin{tabular}{|c|c|c|c|c|c|c|}
\hline & \multicolumn{2}{|c|}{$\mathrm{HE}$} & \multicolumn{2}{|c|}{ BI } & \multicolumn{2}{|c|}{$\mathrm{HO}$} \\
\hline & $\overline{\boldsymbol{x}}$ & $\%$ & $\bar{x}$ & $\%$ & $\bar{x}$ & $\%$ \\
\hline Evitar Grupo de Risco & 0,33 & 28,0 & 0,37 & 37,5 & 0,36 & 13,3 \\
\hline Preservativo peniano & ${ }^{\star} 0,28$ & 28,1 & 0,62 & 62,5 & 0,45 & 45,5 \\
\hline Parceiro único & 0,24 & 24,7 & & & 0,12 & 12,1 \\
\hline Seringa descartável & 0,11 & 11,2 & 0,12 & 12,5 & 0,15 & 15,2 \\
\hline Não sabe & 0,10 & 10,1 & 0,12 & 12,5 & 0,09 & 9,1 \\
\hline Sangue aprovado & 0,06 & 6,7 & & & 0,09 & 9,1 \\
\hline Rezar/não tem jeito & ${ }^{\star *} 0,06$ & 6,7 & & & & \\
\hline Informar-se & 0,04 & 4,5 & & & 0,09 & 9,1 \\
\hline Abstinência sexual & 0,02 & 2,2 & & & 0,18 & 18,2 \\
\hline Higiene & 0,01 & 1,1 & & & & \\
\hline Evitar sintomáticos & & & ${ }^{\star * \star} 0,12$ & 12,5 & 0,09 & 9,1 \\
\hline Evitar esperma & & & ${ }^{* \star \star *} 0,12$ & 12,5 & 0,09 & 9,1 \\
\hline Outros & 0,32 & 32,6 & & & 0,15 & 15,2 \\
\hline
\end{tabular}

TABELA 10. Justificativas dos que Discordaram em se Submeter ao anti-HIV

\begin{tabular}{lrrrrrr}
\hline \hline & \multicolumn{2}{c}{ HE } & \multicolumn{2}{c}{ BI } & \multicolumn{2}{c}{ HO } \\
& \multicolumn{1}{c}{$\bar{x}$} & $\%$ & $\bar{x}$ & $\%$ & $\bar{x}$ & \multicolumn{1}{c}{$\%$} \\
\hline Sabe não estar infectado & 0,14 & 14,6 & 0,01 & 12,5 & 0,15 & 15,1 \\
Não teria coragem & 0,04 & 4,5 & & & 0,12 & 12,1 \\
Teste transmite AIDS & 0,03 & 3,4 & & & 0,03 & 3,0 \\
Não adiantaria & 0,01 & 1,1 & & & 0,06 & 6,1 \\
Só com sintoma & 0,01 & 1,1 & & & 0,03 & 12,1 \\
Prefere evitar & 0,01 & 1,1 & & & 0,06 & 6,1 \\
\hline \hline
\end{tabular}


Em relação aos conteúdos de identidade sexual, valores e participação social, os resultados foram os seguintes: as respostas de identidade sexual foram agrupadas em três tipos: conduta sexual (HE, 43,8\%; BI, 37,5\%; HO,
$51,1 \%$ ); normas (HE, 25,8\%; BI, 25\%; HO, $33,3 \%$ ) e não sabe (HE, 4,5\%; BI, 12,5\%), sendo que $39,3 \%, 25 \%$ e $3 \%$ dos HE, BI e HO, respectivamente, não especificaram os conteúdos (Tabelas 11 e 12).

TABELA 11. Valores de Grupos Sexuais

\begin{tabular}{|c|c|c|c|c|c|c|}
\hline & \multicolumn{2}{|c|}{ HE } & \multicolumn{2}{|c|}{ BI } & \multicolumn{2}{|c|}{ HO } \\
\hline & $\bar{x}$ & $\%$ & $\bar{x}$ & $\%$ & $\bar{x}$ & $\%$ \\
\hline Família & 5,46 & 96,6 & 4,62 & 77,5 & 4,87 & 93,9 \\
\hline Estudos & ${ }^{* *} 5,24$ & 95,5 & 4,50 & 77,5 & 4,39 & 90,9 \\
\hline Carreira profissional & 5,07 & 95,5 & 4,50 & 77,5 & 4,78 & 90,9 \\
\hline Atividade sexual & ${ }^{* * *} 5,03$ & 95,5 & 4,62 & 77,5 & 4,18 & 93,9 \\
\hline Vida afetiva & 3,43 & 94,4 & 4,87 & 77,5 & 5,09 & 97,0 \\
\hline Opinião dos outros & 3,37 & 94,4 & 3,37 & 77,5 & 3,60 & 90,9 \\
\hline Normas esperadas & 3,06 & 86,5 & 3,00 & 77,5 & 3,18 & 90,9 \\
\hline
\end{tabular}

TABELA 12. Formas de Participação Social de Grupos Sexuais

\begin{tabular}{|c|c|c|c|c|c|c|}
\hline & \multicolumn{2}{|c|}{ HE } & \multicolumn{2}{|c|}{ BI } & \multicolumn{2}{|c|}{$\mathrm{HO}$} \\
\hline & $\bar{x}$ & $\%$ & $\bar{x}$ & $\%$ & $\bar{x}$ & $\%$ \\
\hline Individual & 0,60 & 52,8 & 0,37 & 37,5 & 0,45 & 45,5 \\
\hline Religiosa & ${ }^{*} 0,48$ & 28,0 & 0,12 & 12,5 & 0,06 & 6,1 \\
\hline Recreativa & 0,21 & 21,3 & 0,25 & 25,0 & 0,18 & 18,2 \\
\hline Vizinhança & 0,03 & 3,4 & & & 0,03 & 3,0 \\
\hline Político-sindical & ${ }^{* *} 0,01$ & 1,1 & 0,12 & 12,5 & 0,15 & 15,2 \\
\hline Identidade sexual & & & & & ${ }^{* * \star} 0,15$ & 12,1 \\
\hline Profissional & & & & & $* * * * 09$ & 3,0 \\
\hline Outros & 0,13 & 13,5 & 0,25 & 25,0 & 0,09 & 3,0 \\
\hline
\end{tabular}

Alguns exemplos de conteúdos de identidade sexual podem ser lidos abaixo:

(...) sou normal como qualquer outra, tenho prazeres e pratico minhas relações sexuais

(...)

Simplesmente pelo meu prazer.

Normal.

Acho que nāo tenho (identidade).

Creio ser um homossexual (...) discreto e que aceita essa condiçāo, mesmo nāo me expondo para familiares e para a sociedade heterossexual.

Abaixo, apresentamos algumas reações pessoais esperadas diante da AIDS, bem como percepções de relevância social da AIDS e meios e locais considerados adequados para a veiculação de mensagens sobre o assunto (Tabelas 13 a 16). 
TABELA 13. Reações Pessoais Esperadas se um Amigo se Infectasse com o HIV

\begin{tabular}{llllllll}
\hline \hline & \multicolumn{2}{c}{ HE } & \multicolumn{2}{c}{ BI } & \multicolumn{2}{c}{ HO } \\
& $\bar{x}$ & $\%$ & $\bar{x}$ & $\%$ & $\bar{x}$ & $\%$ \\
\hline Apoio & 0,66 & 56,1 & 0,87 & 87,5 & 0,33 & 72,7 \\
Trataria normalmente & 0,20 & 20,2 & & & 0,18 & 18,2 \\
Afastaria-se & 0,12 & 12,4 & 0,12 & 12,5 & & \\
Não sabe & 0,08 & 9,0 & & & 0,09 & 9,1 \\
Nada faria & 0,06 & 6,7 & & & 0,06 & 6,1 \\
Não faria sexo c/ ele(a) & 0,06 & 6,7 & 0,12 & 12,5 & 0,09 & 9,1 \\
Teria crise psicológica & 0,02 & 2,2 & & & 0,06 & 6,1 \\
Outros & 0,04 & 4,5 & & & & & \\
\hline \hline
\end{tabular}

TABELA 14. Reações Pessoais Esperadas Caso se Infectasse com o HIV

\begin{tabular}{lllllll}
\hline \hline & \multicolumn{2}{c}{ HE } & \multicolumn{2}{c}{ BI } & \multicolumn{2}{c}{ HO } \\
& $\bar{x}$ & $\%$ & $\bar{x}$ & $\%$ & $\bar{x}$ & $\%$ \\
\hline Não sabe & 0,41 & 40,0 & 0,12 & 12,5 & 0,36 & 33,3 \\
Se conformaria & 0,34 & 32,5 & 0,62 & 50,0 & 0,16 & 45,5 \\
Teria crise psicológica & 0,28 & 26,9 & 0,25 & 25,0 & 0,42 & 39,4 \\
Se isolaria & 0,10 & 7,8 & 0,12 & 12,5 & 0,06 & 6,1 \\
Não contaminaria outro(a) & 0,07 & 6,7 & & & 0,12 & 12,1 \\
Procuraria ajuda & 0,03 & 3,4 & 0,12 & 12,5 & & \\
Se arrependeria & 0,02 & 2,2 & & & 0,06 & 6,1 \\
Outros & 0,08 & 9,0 & 0,12 & 12,5 & 0,06 & 6,1 \\
\hline \hline
\end{tabular}

* $\mathrm{p} \leq 0,035$

TABELA 15. Relevância Social da Epidemia de AIDS

\begin{tabular}{llllllr}
\hline \hline & \multicolumn{2}{c}{ HE } & \multicolumn{2}{c}{ BI } & \multicolumn{2}{c}{ HO } \\
& $\overline{\boldsymbol{x}}$ & $\%$ & $\bar{x}$ & $\%$ & $\bar{x}$ & $\%$ \\
\hline Existe epidemia & 0,60 & 60,6 & 0,62 & 62,5 & 0,66 & 66,7 \\
A epidemia é mais grave & 0,16 & 14,6 & & & 0,24 & 21,2 \\
A imprensa exagera & 0,12 & 12,4 & 0,25 & 25,0 & 0,12 & 12,1 \\
Não sabe & 0,04 & 4,5 & & & 0,06 & 6,1 \\
Exagero para contenção sexual & 0,01 & 1,1 & & & 0,12 & 12,1 \\
Deve-se exagerar mais & 0,01 & 1,1 & & & 0,03 & 3,0 \\
Omitem fatos & & & & & 0,06 & 6,1 \\
Outros & 0,17 & 18,0 & 0,12 & 12,5 & 0,12 & 12,1 \\
\hline \hline
\end{tabular}

$* \mathrm{p} \leq 0,017 ; \quad$ ** $\mathrm{p} \leq 0,052$ 
TABELA 16. Meios e Locais de Informação Considerados Adequados para Informar sobre a AIDS

\begin{tabular}{|c|c|c|c|c|c|c|}
\hline & \multicolumn{2}{|c|}{$\mathrm{HE}$} & \multicolumn{2}{|c|}{$\mathrm{BI}$} & \multicolumn{2}{|c|}{ HO } \\
\hline & $\bar{x}$ & $\%$ & $\bar{x}$ & $\%$ & $\bar{x}$ & $\%$ \\
\hline Locais públicos & 0,45 & 37,1 & 0,37 & 37,5 & 0,69 & 45,3 \\
\hline Todos os meios & 0,40 & 38,2 & 0,62 & 62,5 & 0,54 & 48,5 \\
\hline Rádio e TV & ${ }^{*} 0,20$ & 16,9 & 0,12 & 12,5 & 0,60 & 39,4 \\
\hline Grupos de Risco & 0,14 & 12,3 & & & 0,15 & 12,1 \\
\hline Família/individual & 0,10 & 10,1 & 0,25 & 25,0 & 0,09 & 9,1 \\
\hline Material escrito & ${ }^{* *} 0,08$ & 6,7 & & & 0,16 & 40,3 \\
\hline Não deve ser veiculada & 0,03 & 3,4 & 0,12 & 12,5 & & \\
\hline Trabalho/sindicato & $* * 0,02$ & 2,2 & 0,25 & 25,0 & 0,09 & 6,0 \\
\hline Igreja/grupo jovem & 0,01 & 1,1 & 0,12 & 12,5 & 0,06 & 3,0 \\
\hline Outros & 0,30 & 29,2 & 0,25 & 25,0 & 0,27 & 15,1 \\
\hline
\end{tabular}

Conforme se pode ler na Tabela 1 , houve concentração de respostas em relação a algumas formas de contágio conhecidas, mas a idéia de contágio parece ter feito lembrar outros temas do repertório dos sujeitos nesse assunto. Assim, as formas mais comuns de contágio para os grupos se referiram à relação sexual, ao sangue, à droga injetável, notando-se que entre os HO houve certa tendência de apresentar respostas sangue.

Quanto aos grupos vulneráveis ao vírus, os grupos estudados tenderam a generalizar a todos a possibilidade de contágio (Tabela 2).

As formas de observar os sintomas da doença mais referidas foram alterações corporais externas, entre $\mathrm{HE}$ e $\mathrm{HO}$, seguidas, para os mesmos sujeitos, por alterações corporais internas e infecções. Porém, os HO e BI ressaltaram ainda mais infecções que os demais grupos (Tabela 3).

$O$ conhecimento a respeito da origem da AIDS não suscitou, entre os grupos, muitas respostas, as quais foram não-especificas (outros) ou não sabe. Contudo, devemos ressaltar a maior ênfase atribuída pelos HO à idéia de que a AIDS surgiu no laboratório (Tabela 4).

Em termos de conhecimento sobre tratamentos disponíveis ao portador do vírus da AIDS, os $\mathrm{HO}$ os consideraram, mais que os $\mathrm{HE}$, paliativos; os BI preferiram dizer que não sabem (Tabela 5).

A leitura da Tabela 6, sobre práticas sexuais declaradas pelos grupos, revela-nos haver diferenças qualitativas entre si. Assim, os HE observados relataram maior prática de sexo vaginal e vaginal e oral com/sem masturbação, ainda que os HE masculinos tenham apontado alguma prática vaginal, oral e anal com/sem masturbação. Mas, entre os $\mathrm{HE}$, outro aspecto importante é a abstinência sexual, tanto para o sexo masculino quanto para o feminino. Já os HO tenderam a se concentrar em sexo oral e anal com/sem masturbação e anal com/sem masturbação, mas houve importante contingente que indicou praticar a masturbação individual. Os BI masculinos, por sua vez, caracterizaram-se por uma dispersão maior entre as práticas, mas concentraram-se em sexo vaginal e oral com/sem masturbação. Aliás, nenhum BI declarou-se em abstinência ou praticando masturbação individual.

Em termos de risco através da atividade sexual, HO e HE foram os grupos mais expostos (Tabelas 7 e 8).

Por outro lado, as estratégias adotadas para evitar o contágio (Tabela 9) mostraram o uso de preservativo como mais importante para $\mathrm{HO}$ e BI, seguidas, para $\mathrm{HO}$, de abstinência sexual e seringa descartável e, para BI, evitar grupos de risco. Já os HE preferiram evitar grupos de risco, seguido por preservativo e parceiro único. Entre os $\mathrm{HO}$ e $\mathrm{BI}$, notou-se tendência de evitar sintomático e evitar esperma.

Quando perguntados se submeteriam-se ao 
teste-AIDS, todos os grupos tenderam a concordar. $\mathrm{E}$ os argumentos obtidos para justificarem a realização do teste se concentraram na idéia de segurança, no que os HO se destacaram mais.

Os argumentos apresentados pelos grupos para não se submeterem ao teste-AIDS foram basicamente de dois tipos: sabe que não está infectado e não teria coragem (Tabela 10).

No que toca à identidade sexual representada, os HO e HE se caracterizaram por dar mais ênfase à conduta sexual, dando menos importância que os BI aos aspectos normativos. Aliás, nas respostas dos HE, foram os sujeitos masculinos os que indicaram relativamente mais experiência e conteúdo verbal a partir da pergunta que fizemos.

Em termos valorativos, os $\mathrm{HE}$ deram mais importância, em ordem decrescente, à família, estudos, carreira profissional e atividade sexual, enquanto que $\mathrm{BI}$, à vida afetiva, atividade sexual, família, carreira profissional e estudos, e os HO, por sua vez, à vida afetiva, família, carreira profissional, estudos e atividade sexual. É bom destacar que os HE apresentaram valorização maior para a família, estudo e atividade sexual (Tabela 11).

Para os três grupos investigados, a forma de participação social que denominamos individual mostrou-se a mais frequiente, seguida, no caso dos $\mathrm{HE}$, por religiosa, recreativa; para $\mathrm{BI}$, recreativa, religiosa, político-sindical, identidade sexual; e para $\mathrm{HO}$, além de recreativa e político-sindical, identidade sexual e menos religiosa que os demais grupos. Contudo, os HE indicaram relativamente maior participação religiosa, e os $\mathrm{HO}$, político-sindical, identidade sexual e profissional (Tabela 12).

As reaçōes pessoais esperadas face a um amigo infectado se concentraram em apoio e trataria normalmente. Ainda assim, um certo número de $\mathrm{HE}$ e $\mathrm{BI}$ disse que se afastaria de um amigo portador do vírus (Tabela 13).

No caso de se descobrirem infectados, por sua vez, as representações coletadas se concentraram basicamente em não sabe, se conformaria e crise psicológica, ainda que com tendência variada entre os grupos: BI e HO se conformariam e crise psicológica, e HE, não sabe, se conformaria e crise psicológica (Tabela 14).
Em relação às repercussões sociais do vírus, também houve consenso em achar que existe epidemia, mas alguns sujeitos dos grupos HE e HO consideraram que a epidemia é ainda mais grave, enquanto que os $\mathrm{BI}$, por seu turno, manifestaram pensamento de que há exagero da imprensa, e os $\mathrm{HO}$, de que tal exageração é para conter a sexualidade, embora sem diferença significativa estatisticamente (Tabela 15).

Os três grupos observados destacaram todos os meios, locais públicos e rádio e TV como formas principais de veiculação de informação sobre a AIDS. Além disso, os HE e HO apontaram locais de grupos de risco em terceiro e quarto lugares, respectivamente; os $\mathrm{BI}$, trabalho e sindicato como outros locais e meios também válidos; e os HO, material escrito (Tabela 16).

\section{DISCUSSÃO}

O instrumento que elaboramos e aplicamos pretendia, numa primeira investigação, fixar alguns parâmetros psicossociais a fim de entendermos melhor alguns aspectos ligados ao fenômeno da AIDS. De modo geral, os grupos estudados apresentaram um nível de informação a respeito da AIDS insuficiente, impreciso, ainda que diferenciado intergrupalmente. De todo jeito, parece que o que se reteve de campanhas de informação foram alguns slogans genéricos, aparecendo, ainda que menos frequilentemente, crenças antigas, como a que considera os objetos pessoais transmissores do vírus (Jodelet, 1989).

Os grupos estudados concordaram com relação a outros aspectos da representação da AIDS, tal como o que estende a todos a possibilidade de contágio, mas é bom ressaltar que HO e BI consideraram que o vírus foi gerado no laboratório. Poderfamos nos perguntar se tal representação não estaria ligada à situação de relativa marginalidade desses grupos sexuais em relação às instituições sociais, configurando uma relação defensiva com as mesmas. Se tais comportamentos existiam antes da epidemiaAIDS, eles teriam sido reativados pela exposição da identidade homossexual ligada à doença (Mott, 1991; Tosta, 1991).

Cabe-nos ainda mencionar que os resultados 
indicaram alguma diferença quanto ao modo de conhecer os sintomas da AIDS adotados por $\mathrm{HO}$, que tenderam a incluir mais o item infecçōes, refletindo provavelmente experiências e posturas informativas mais especificas. Um outro exemplo da mesma tendência é a representação, entre os HO, de que existem tratamentos paliativos para a AIDS, de conotação algo mais positiva que sem tratamento, mais comum entre os HE.

As práticas sexuais dos HE indicaram pouca ou quase nenhuma declaração de sexo anal exclusivo ou combinado a outras práticas. Por outro lado, a abstinência sexual observada poderia ser melhor compreendida a partir de uma leitura da distribuição desse grupo entre as faixas etárias: enquanto $66,3 \%$ dos $\mathrm{HE}$ estavam entre 15 e 21 anos de idade, os BI e HO situavam-se acima de 22 anos em $87,5 \%$ e $87,9 \%$, respectivamente. Contudo, o estudo de Henning \& Tamayo (1989) mostrou que o nivel de conhecimento sobre a AIDS não só era menor entre os que praticavam menos sexo, mas também poderia justificar psicologicamente um retardo do desenvolvimento da vida sexual ativa.

Entre os HO estudados, $26,6 \%$ se declararam praticando, ainda que em menor número, a masturbação exclusiva ou combinada a outras formas, possivelmente como reação à epidemia. Se atentarmos para os índices de risco representados por HE e HO, estes constituiam-se, à época do estudo, em grupos com maior número de parceiros e de vezes que haviam praticado sexo sem preservativo, situando-se, portanto, em condições epidemiológicas distintas. Contudo, as estratégias adotadas para se evitar o vírus foram também diferenciadas. Ou seja, os HO, sendo os mais atingidos, produziram um padrão de resposta que privilegiou o preservativo, seguido de abstinência sexual. Esta última tendência $(18,2 \%)$, embora menor, pode significar uma crise psicológica importante e aumentar a vulnerabilidade a práticas sexuais de risco, conforme constatou Prieur (1990) ao estudar HO que as aceitavam para não perder a oportunidade de viver um romance, na expectativa de não lhes ser muito frequientes e/ou após periodos de privação, reais ou simbolizados. Neste sentido, o mesmo grupo tem na prática anal uma dimensão importante da sua identidade sexual, ameaçada pela AIDS e ainda em 1989 não contornada com o uso do preservativo. Neste caso, a abstinência e a masturbação individual parecem ser mecanismos de negação ou evitação do problema que ampliam ainda mais as dificuldades de realização afetiva e de reconhecimento social vividas por membros deste grupo.

O grupo BI masculino, por sua vez, apesar de muito reduzido em nossa amostra, apresentou maior variedade de práticas sexuais, o que mereceria um estudo posterior, pois trata-se de um dos elos das cadeias de transmissão do vírus para os grupos HE e HO.

Por outro lado, o grupo HE feminino revelouse ligado às idéias de evitar grupos de risco e ter parceiro único, que são, evidentemente, simplificações, ou mesmo deslocamentos de atenção, para evitar enfrentar o problema realisticamente na vida do casal, possivelmente temendo abalar a confiança mútua ou a quantidade de prazer vivido. Estas representaçōes revelam-se insuficientes em termos preventivos (Bauman \& Siegel, 1987), sustentando-se, em parte, numa informação superficial da síndrome, mas também, em função de valores e ideologias sobre a familia, de origens remotas (LéviStrauss, 1966), ganhando atualidade diante de uma epidemia inicialmente considerada de sexualidades desviantes (homossexual, promiscua) ou de consumo de drogas ilegais, sobretudo em meios sociais conservadores (Sontag, 1989).

Pudemos constatar o quão era centrada a definição de identidade sexual de $\mathrm{HE}$ e $\mathrm{HO} \mathrm{em}$ termos de conduta sexual (ou seja, desejo, prática sexual). Contudo, esses grupos estavam sob influência valorativa de agentes sociais diferentes: enquanto os HE viviam na órbita da familia, estudos, vida religiosa e meios de comunicação de massa, os HO eram também ligados ao grupo de identidade sexual, via práticas sexuais e valorização da vida afetiva. Já os BI tendiam para uma posição sócio-cultural mista, procurando valorizar a vida recreativa, práticas sexuais, mas também a identidade sexual em termos normativos, vida religiosa. Daf porque os HE tenham situado a atividade sexual em quarto lugar de importancia, os BI, em segundo lugar, após vida afetiva, e os HO, em quinto, após vida afetiva, familia, carreira, 
estudos, em ordem decrescente. No caso dos HE, estes cursavam o secundário, com perspectivas de entrada no mercado de trabalho ou de prosseguir estudando em curso superior. $O$ grupo $\mathrm{HO}$, por sua vez, $50 \%$ dos quais com instrução universitária, parece-nos mais estabilizado valorativamente.

Ainda que tenha havido uniformidade entre os grupos quanto a reagir com apoio e tratando normalmente um amigo contaminado, houve certa tendência dos HE e BI ao afastamento. Tal reação poderia prejudicar um possivel papel no tratamento de portadores do vírus da AIDS, conforme se demonstrou em um estudo feito em Nova Iorque com soropositivos, os quais, em função de se representarem contando com apoio social ou não, retardaram significativamente o desenvolvimento de sintomas da doença posteriormente (Zich \& Temoshok, 1987). Houve manifestação verbal de cuidado social entre os HO que estudamos, que procurariam não contaminar outros caso se tornassem portadores do vírus.

Parece ter existido certa relação entre o fato de representar a relevância social da epidemia e o tipo de reação esperada caso se contaminasse. Assim, os grupos consideraram basicamente que existe epidemia e que é mais grave e, simultaneamente, não sabem como reagiriam e se conformariam, seguidas por teriam crise psicológica. Contudo, se conformariam, entre HO e BI, indica certa preparação para o problema, ainda que os BI tenham se destacado em considerar que a imprensa exagera, e os $\mathrm{HO}$, que deve-se exagerar mais.

Uma forma de entender a representação da gravidade do problema considerado é relacionálo aos hábitos de informação ou ao grau de risco representado/vivido. Assim, o grupo HO se sobressaju ao preferir material escrito sobre o assunto, ou seja, mais preciso; os demais grupos optaram por locais e meios usualmente veiculadores de informação menos elaborada e de consumo rápido. A representação de que todos estão sob ameaça combinou-se à preferência por formas de comunicação de massa. Por outro lado, os BI reclamaram um tratamento mais privado e específico aos grupos profissionais.

O estudo acima relatado procurou rastrear parâmetros psicossociais envolvidos na problemática da AIDS. Ele serviu-nos, sobretudo, para fazer um mapeamento qualitativo de temas importantes existentes no repertório simbólico dos grupos observados, a serem aprofundados em investigações ulteriores.

\section{AGRADECIMENTOS}

Agradecemos a colaboração de Helena Olivetto Greco e ao Colégio JK, da Asa Norte (Brasilia), por terem interrompido as suas atividades para que este estudo fosse realizado; ao Centro de Processamento de Dados da UnB e ao $\mathrm{CNPq}$, que auxiliaram no tratamento estatistico e no financiamento deste trabalho, respectivamente.

\section{RESUMO}

SOUZA-FILHO, E. A. \& HENNING, M. G. Representações Sociais da AIDS, Práticas Sexuais e Vida Social entre Heterossexuais, Bissexuais e Homossexuais em Brasília, Brasil. Cad. Saúde Públ., Rio de Janeiro, 8 (4): 428-441, out/dez, 1992.

Através de 33 questões abertas e fechadas, observamos as representações sociais da AIDS, práticas sexuais e preventivas, autorepresentações, vida social, valores e meios de informaçōes de 89 heterossexuais (HE), 7 bissexuais (BD e 33 homossexuais (HO). Os resultados mostraram uma crença geral de que todos estão vulneráveis ao vírus. Os $\mathrm{HE}$ e HO consideraram alterações corporais externas como o principal sintoma da AIDS. Os HE praticavam mais sexo vaginal e oral $(52,69 \%)$, os HO, sexo anal e oral $(43,33 \%)$, e os BI se dispersaram entre muitas práticas sexuais. Além do uso de preservativo, os $\mathrm{HE}$ evitariam grupos de risco e prefeririam um parceiro único, enquanto os HO evitariam praticar sexo $(18,2 \%)$.

Apesar das auto-representações de HE e HO em termos de conduta sexual, estes valorizaram mais a familia do que a atividade sexual - o que pode ser um sinal de mudança psicossocial. 
Algumas sugestões de pesquisa foram feitas sobre a prática BI como elo entre os grupos, sobre as relaçōes de poder e confiança entre parceiros, e sobre a urgência de novos estudos a respeito da vida psicossocial desses grupos.

Palavras-Chave: AIDS; Representação Social; Comportamento Sexual; Psicologia Social

\section{REFERÊNCIAS BIBLIOGRÁFICAS}

ADEODATO, S., 1991. AIDS se alastra entre os brasileiros mais pobres. Jornal do Brasil, (Rio de Janeiro) 17 de março, p. 22.

BAUMAN, L. J. \& SIEGEL, K., 1987. Misperception among gay men of the risk for AIDS associated with the sexual behavior. Journal of Applied Social Psychology, 17: 329-350.

ECHABE, A. E. \& ROVIRA, D. P., 1989. Social representations and memory. European Journal of Psychology, 19: 543-55.

FRY, P., 1982. Para inglês ver - identidade $e$ política na cultura brasileira. Rio de Janeiro: Zahar.

GUNTHER, H.; TRÓCCOLI, B. T. \& KELLER, M. L., 1991. AIDS e adolescentes no Brasil: disposição para prevenção. Comunicação apresentada na mesa-redonda AIDS na Universidade, Instituto de Psicologia da Universidade de Brasilia. (Mimeo)

HENNING, M. G. \& TAMAYO, A., 1989. Relação entre o nivel de conhecimento a respeito da AIDS e o comportamento sexual. In: XIX Reunião da Sociedade de Psicologia de Ribeirão Preto, Resumos, p. 144, Ribeirāo Preto.

HERZLICH, C., 1972. La représentation sociale. In: Introduction da la Psychologie Sociale (S. Moscovici, org.), vol 1, pp. 303-325, Paris: Larousse.

HERZLICH, C. \& PIERRET, J., 1988. Une maladie dans l'espace publique. Le Sida dans six quotidiens français. Annales-Esc, 5: 1109-1134.

JODELET, D., 1989. Folies et représentations sociales. Paris: P. U. F.

LÉVI-STRAUSS, C. 1966. A Familia. In: Homem, Cultura e Sociedade (H. L. Shapiro, org.), pp. 308-333, Rio de Janeiro: Fundo de Cultura.

MARKOVA, I. \& WILKIE, P., 1987. Representations, concepts and social change: the phenomenon of AIDS. Journal for the Theory of Social Behaviour, 15: 389-409.
MARTIN, D.; CASTRO-FILHO, D. \& ARAÚJO, G. R. S. de, 1991. Comportamento sexual e anticonceptivo em São Paulo. São Paulo: Gerd Wenzel Eventos.

MOSCOVICI, S., 1978. A representação social da Psicanálise. Rio de Janeiro: Zahar.

MOTT, 1., 1991. Pessoa com AIDS: os soropositivos e "aidéticos", uma nova minoria social. Comunicação apresentada no Encontro AIDS: repercussóes psicossociais, Instituto de Psicologia da Universidade de São Paulo. (Mimeo)

PARKER, R., 1987. Acquired Immunodeficiency Syndrome in Urban Brazil. Medical Anthropology Quarterly, 1: 155-175.

PERLONGHER, N., 1991. O desaparecimento da sexualidade. Comunicação apresentada no AIDS: repercussões psicossociais, Instituto de Psicologia da Universidade de Sáo Paulo. (Mimeo)

POLLAK, M., 1987. A homossexualidade masculina ou a felicidade no gueto? In: Sexualidades Ocidentais (P. Ariès \& A. Béjin, orgs.), pp. 54 76, São Paulo: Brasiliense.

POLLAK, M., 1987. L'identité sociale et gestion d'un risque de santé - les homosexuels au SIDA. Actes de la Recherche en Sciences Sociales, 68: 77-102.

PRIEUR, A., 1990. Norwegian gay men: reasons for continued practice on unsafe sex. Aids Education and Prevention, 2: 109-115.

TOSTA, R. M., 1991. As práticas e vivências homossexuais masculinas frente à AIDS; novas formas de normalização sexual. Comunicação apresentada no AIDS: repercussőes psicossociais, Instituto de Psicologia da Universidade de São Paulo. (Mimeo)

SOUZA-FILHO, E. A., 1992. Representaçōes sociais da AIDS, práticas sexuais e preventivas entre heterossexuais, bissexuais e homossexuais no Rio de Janeiro e em Brasilia. Relatório Bianual, Brasilia: Conselho Nacional de Desenvolvimento Cientifico e Tecnológico.

SONTAG, S., 1990. A AIDS e suas Metáforas. São Paulo: Companhia das Letras.

TOURAINE, A., 1984. Les mouvements sociaux: objet particulier ou problème central de l'analyse sociologique. Revue Francaise de Sociologie, 25: 3-19.

ZICH, J. \& TEMOSHOK, L., 1987. Perceptions of social support in men with AIDS and ARC: relationships with distress and hardiness. Journal of Applied Social Psychology, 17: 193-215. 